\title{
Active transforming growth factor- $\beta$ is associated with phenotypic changes in granulomas after drug treatment in pulmonary tuberculosis
}

Robert M. DiFazio', Joshua T. Mattila², Edwin C. Klein ${ }^{3}$, Lauren R. Cirrincione ${ }^{4}$, Mondraya Howard', Eileen A. Wong ${ }^{1}$ and JoAnne L. Flynn ${ }^{1 *}$

\begin{abstract}
Background: Tuberculosis (TB) chemotherapy clears bacterial burden in the lungs of patients and allows the tuberculous lesions to heal through a fibrotic process. The healing process leaves pulmonary scar tissue that can impair lung function. The goal of this study was to identify fibrotic mediators as a stepping-stone to begin exploring mechanisms of tissue repair in TB.

Methods: Hematoxylin and eosin staining and Masson's trichrome stain were utilized to determine levels of collagenization in tuberculous granulomas from non-human primates. Immunohistochemistry was then employed to further interrogate these granulomas for markers associated with fibrogenesis, including transforming growth factor- $\beta$ (TGF $\beta$ ), a-smooth muscle actin (aSMA), phosphorylated SMAD-2/3, and CD163. These markers were compared across states of drug treatment using one-way ANOVA, and Pearson's test was used to determine the association of these markers with one another.

Results: TGF $\beta$ and ASMA were present in granulomas from primates with active TB disease. These molecules were reduced in abundance after TB chemotherapy. Phosphorylated SMAD-2/3, a signaling intermediate of TGF $\beta$, was observed in greater amounts after 1 month of drug treatment than in active disease, suggesting that this particular pathway is blocked in active disease. Collagen production during tissue repair is strongly associated with TGF $\beta$ in this model, but not with CD163+ macrophages.

Conclusions: Tissue repair and fibrosis in TB that occurs during drug treatment is associated with active TGF $\beta$ that is produced during active disease. Further work will identify mechanisms of fibrosis and work towards mitigating lung impairment with treatments that target those mechanisms.
\end{abstract}

Keywords: Tuberculosis, Drug treatment, Transforming growth factor- $\beta$, Collagen I

\section{Background}

With eight million new cases and 1.5 million deaths annually worldwide, tuberculosis (TB) is one of the humanity's greatest health threats [1]. Granulomas, the pathologic hallmark of TB, are well-circumscribed organized collections of host immune cells that form in

\footnotetext{
*Correspondence: joanne@pitt.edu

'Department of Microbiology and Molecular Genetics, University of Pittsburgh School of Medicine, Pittsburgh, PA 15261, USA

Full list of author information is available at the end of the article
}

response to the inhalation of aerosols containing $\mathrm{Myco}$ bacterium tuberculosis (Mtb)-the causative agent of TB. Although granulomas can function to kill or contain Mtb, they can also serve as a niche for growth and persistence of the organism [2-4]. Granulomas often feature a necrotic center and are thus dubbed as necrotizing or caseous, while granulomas lacking this necrosis are said to be non-necrotizing. Granulomas feature epithelioid macrophages, elongated cells with larger nuclei, surrounded by other macrophages and lymphocytes [5]. Bacteria can be found in epithelioid macrophages and in 
caseum [5]. Uncontrolled replication leads to dissemination of the bacteria and formation of new granulomas. However, some granulomas can restrain bacterial dissemination or even develop locally sterilizing immunity. As a result, these granulomas are often fibrotic and can contain a calcified core (referred to as fibrocalcific lesions) [6]. A mixture of necrotizing and collagenous lesions is typical of the secondary pulmonary tuberculosis and is referred to as fibrocaseous disease [6,7]. Although the host factors that lead to control or dissemination of a single granuloma are unclear, we have demonstrated that various types of granulomas and outcomes exist within a single nonhuman primate, similar to humans [6, 8].

Tissue fibrosis can result from a wound healing response that includes fibroblast activation and recruitment, production of extracellular matrix materials, and distortion of the normal tissue architecture. The most common extracellular matrix component is collagen I, which is the most fibrous form of collagen and represents about $84 \%$ of the collagen produced by fibroblasts [9]. Fibrosis can be caused by a local inflammatory response, and fibrosisrelated pathogenesis is associated with dysfunction of many organs, including lungs, liver, and kidneys [10-12]. Transforming growth factor- $\beta$ (TGF $\beta$ ) is the main cytokine implicated in fibrogenesis, although other cytokines are implicated, including TNF, IL-6, IL-10, IL-13, and IL17 [13-18]. TGF $\beta$ is produced in a latent form (L-TGF $\beta$ ) and can be activated through the plasmin protease pathway, CD36 and thrombospondin (TSP), reactive oxygen and nitrogen species, hypoxia, low $\mathrm{pH}$, and matrix metalloproteases [19]. Active TGF $\beta$ utilizes type 1 and 2 TGF $\beta$ receptors, signaling through a variety of intermediaries, including phosphorylated SMAD-2/3 [20]. Through these intermediaries, TGF $\beta$ stimulates differentiation of fibroblasts into myofibroblasts that then produce alphasmooth muscle actin ( $\alpha$ SMA), a key indicator of and contributor to fibrotic pathogenesis [21]. TGF $\beta$ has been observed and measured in pulmonary fibrosis and, in the lung, is produced by alveolar macrophages, fibrocytes, and lung epithelial cells [22-25]. Alveolar macrophages from humans with pulmonary fibrosis display an alternatively activated (M2) phenotype, and induction and maintenance of M2 macrophages is critical to pathology in pulmonary fibrosis [26]. M2 macrophages are also major producers and activators of TGF $\beta$ [24].

Although several types of pulmonary fibrosis have been characterized and studied, fibrosis in tuberculosis is not well-understood. Significant pulmonary impairment was observed in $59 \%$ of patients with TB disease [27], half of whom had less than $50 \%$ of their original forced vital capacity [27]. This loss of pulmonary function resulted in 177 subjects losing 1189 disability adjusted-life years [28]. Lung function does not improve over the course of chemotherapy [29], and this chronic impairment increases incrementally with the number of TB episodes experienced in a progressive manner [29]. The main course of treatment for post-tuberculosis lung damage is pulmonary rehabilitation, which has mixed results [30], highlighting the need for more targeted therapies to resolve TB-induced fibrosis and scarring. Since macrophages produce TGF $\beta$ in pulmonary fibrosis, and are a major cellular component of granulomas [24], macrophages may be important contributors to fibrosis in $\mathrm{TB}$ lesions [31]. The environment of the granuloma may contain almost all of the conditions that activate TGF $\beta$, including hypoxia [32], nitrogen radicals [5], and metalloproteases [33], so it is likely that the disease process activates TGF $\beta$ locally at the site of infection. Cutaneous TB lesions in humans have been noted as centers of fibrosis, with lesions containing active TGF $\beta$ [34]. Patients with TB have peripheral blood monocytes and alveolar macrophages that produce and active more TGF $\beta$ than cells from healthy controls $[35,36]$. TGF $\beta$ has also been observed directly in granulomas from human TB patients by immunohistochemistry [37].

Drug treatment for M. tuberculosis infection $[38,39]$ is a lengthy process that slowly clears bacterial burden in the lung and induces tissue repair in TB-affected lung. The factors that promote fibrotic resolution of tuberculous granulomas are poorly understood. This has been a challenging topic to address because of difficulties associated with studying human TB and a lack of appropriate mouse models demonstrating the granuloma structures seen in humans. Our laboratory previously published that drugtreated macaques with TB had fibrotic granulomas, and the fibrotic granulomas were most often sterile [40], representing a successful outcome of drug treatment. Since macaques recapitulate the spectrum of granuloma types and infection outcomes seen in humans, they represent a useful system for studying the process of drugassociated fibrosis. Understanding the fibrotic processes that occur in TB may provide insights into treatments to safely resolve residual lung fibrosis during or after drug therapy. The objective of this study was to determine how the cell types and molecules associated with pulmonary fibrosis differ between granulomas associated with active $\mathrm{TB}$ and fibrotic changes after chemotherapy. This study will open up further exploration of the fibrogenic mechanisms, with the aim of developing treatments to minimalize or reverse scarring after drug treatment.

\section{Results}

Macrophages experience spindloid transformation in tuberculous granulomas

After the original uptake of mycobacterial organisms by macrophages, a chemokine and cytokine cascade leads to monocyte recruitment, differentiation of macrophages, 
and epithelioid transformation. The latter is characterized by a large, plumper, more eosinophilic staining cell with somewhat elongated, sole-shaped nuclei (Fig. 1, dashed arrow). In contained tuberculous lesions, especially nonnecrotizing ones that do not expand or infiltrate into adjacent bronchial or alveolar airspaces, further change then occurs globally in the appearance of these cells. Cell bodies become less plump and more streaming in shape, with nuclei progressing to a more tapered, spindled appearance (Fig. 1, solid arrow). A similarity between other aspects of nuclear (e.g., chromatin pattern) and cytoplasmic morphology remains between cells in different stages of this continuum. Fibroblasts and associated collagen fiber formation (Fig. 1, arrowhead) are seen in both necrotizing and non-necrotizing lesions, followed by the development of dense bands and sheets of fibrous connective tissue. Eosinophils have been linked to promotion of fibrogenesis [41] but are rarely abundant in lung granulomas in nonhuman primates (unpublished observations).

\section{Granulomas exhibit a range of collagen deposition before and after drug treatment}

Our laboratory has previously provided histologic evidence for the resolution of TB pathology after 2 months of chemotherapy in cynomolgus macaques [40]. We used Masson's trichrome staining, a histologic technique for specifically identifying collagen, on pooled granulomas from two previously published drug studies [40, 42] to confirm our hematoxylin and eosin (H\&E)-based findings on the same set of animals. We examined if representative lesions have more (Fig. 2a, c, e) or less (Fig. 2b, d, f) collagen present by both histological stains. The more collagenous granuloma from an animal with active disease exhibits a faint ring of peripheral collagen

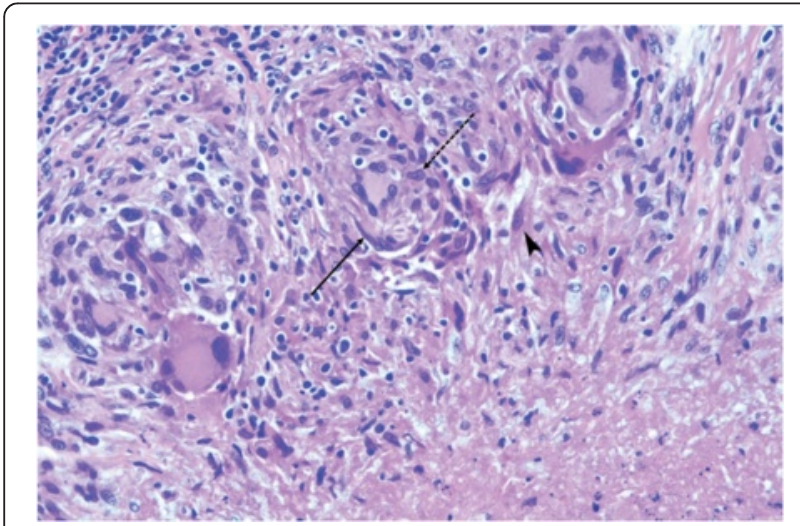

Fig. 1 Macrophages experience spindloid transformation in tuberculous granulomas. H\&E stained granuloma that demonstrates spindloid transformation of histiocytes. This figure provides microhistological examples of: epithelioid transformation of macrophages with elongated nuclei, dashed arrow, top; tapered, spindled macrophages further transformed, solid arrow, left; and collagen deposition, arrowhead, right. Magnification at ×200 deposition (Fig. 2a), which is not uncommon in tuberculous lesions, while less collagen-rich non-fibrotic granulomas lack this peripheral cuff of collagen (Fig. 2b). After 1 month of drug treatment, some granulomas exhibit a "healing" phenotype [40], as evidenced by a thickening of the peripheral fibrotic cuff and central fibrous organization (Fig. 2c). Other granulomas lack this degree of collagenization but still exhibit some central fibrous organization (Fig. 2d). By 2 months of chemotherapy, there are many lesions whose phenotype is solidly collagenous (Fig. 2e). There are still lesions though that are not completely resolved, and these granulomas are characterized as having some central fibrous development without a robust peripheral cuff (Fig. 2f). These data suggest potentially different cellular mechanisms of tissue repair in tuberculosis, with various degrees of collagenization. We then quantified the photomicrographs for aniline blue-stained collagen to determine how drug treatment modifies collagen deposition (Fig. 2g). We found that granulomas from animals with active disease and 1 month of chemotherapy exhibit a wide range of collagen deposition (Fig. 2g). After 2 months of drug therapy, half of the granulomas are highly collagenous, but the other half displayed lesser degrees of collagenization (Fig. 2g). To put these data within the context of bacterial burden and sterilization, we divided the number of sterile lung granulomas by the total number of lung granulomas to get percentages of sterile lung granulomas for all of the animals in the study (Additional file 1: Figure S1). We found that the frequency of sterile granulomas increases as drug therapy progresses (Additional file 1: Figure S1a) and that this rise is concurrent with a significant increase the presence of collagen-rich granulomas $(p=0.0002$; Additional file 1: Figure S1b).

Tuberculous granulomas bear signs of TGF $\beta$-driven fibrosis We next wanted to determine whether M2 macrophages and TGF $\beta$ were associated with tissue repair. Granulomas from animals with active disease, 1 month of antibiotic ( $\mathrm{ABX}$ ) therapy, and 2 months of $\mathrm{ABX}$ were analyzed by multiparameter immunohistochemistry for collagen 1 , phosphorylated (p) SMAD-2/3 (an intermediary of TGF $\beta$ signaling), and CD163 (a marker of M2-polarized macrophages in primates [5]) or TGF $\beta$, L-TGF $\beta$, and aSMA using appropriate controls (Additional file 2: Figure S2). This approach provides information on the location and quantity of factors upstream of TGF $\beta$ induction and TGF $\beta$ activation. We found that caseous granulomas from animals with active, untreated disease contained strongly staining fibrils of collagen I that could be visualized in the central region of the lesion while pSMAD-2/3+ and $\mathrm{CD} 163+$ cells were present along the periphery of the structure (Fig. 3a). In similar granulomas from untreated animals, active TGF $\beta$ was present around the necrotic 

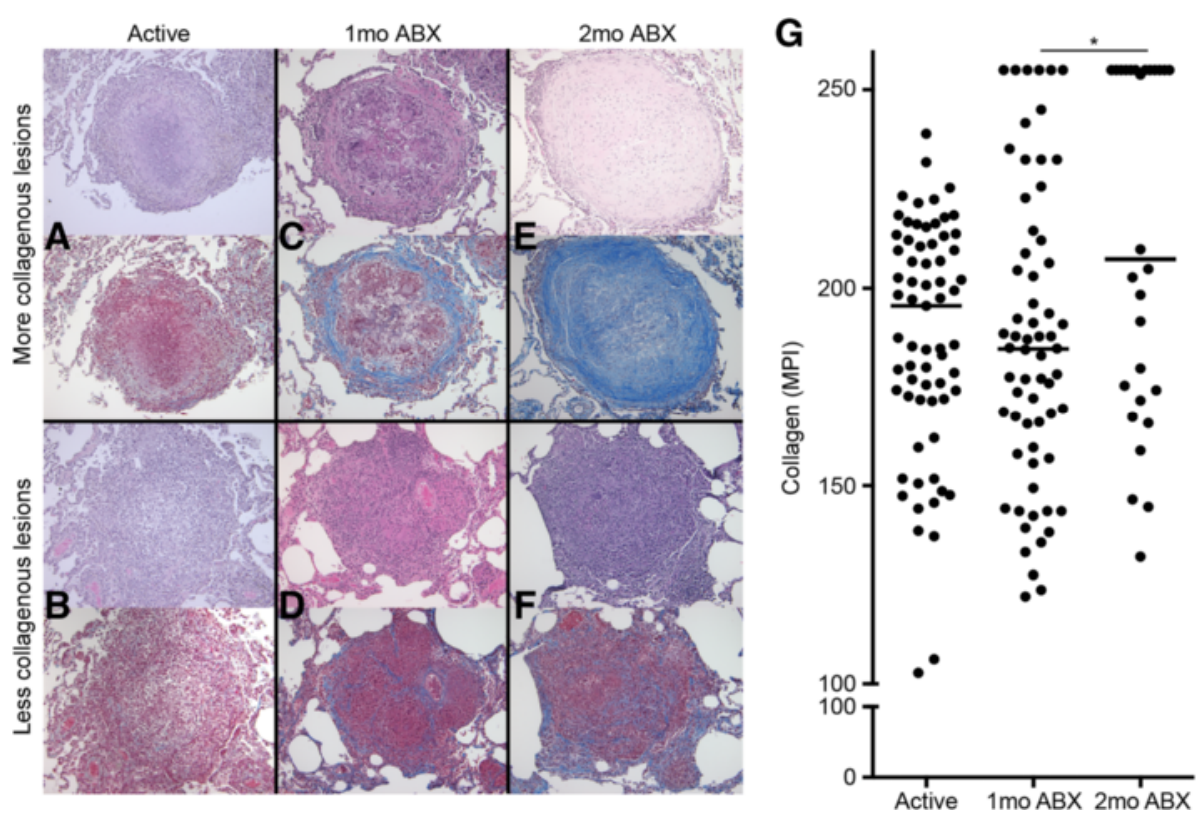

Fig. 2 Granulomas exhibit range of collagen deposition before and after drug treatment. Representative sections of histology from granulomas pooled from two TB chemotherapy studies [40, 42], each with H\&E staining (top panel) and Masson's trichrome staining (bottom panel) for the same lesion. a Granuloma from active disease with some peripheral fibrogenesis. b Granuloma from active disease with no apparent collagenization. c Granuloma from 1-month drug-treated animal with robust peripheral fibrotic cuffing. d Granuloma from 1-month drug-treated animal with some central fibrous organization. e Fibrotic granuloma from 2-month drug-treated animal. f Granuloma from 2-month drug-treated animal with pervasive central fibrous organization. $\mathbf{g}$ Quantification of all Masson's trichrome sections. Median pixel intensity was calculated from the blue channel of each micrograph and plotted by stage of drug therapy. Bars in each column represent medians. Kruskal-Wallis test: $p=0.0285 .{ }^{*} p<0.05$
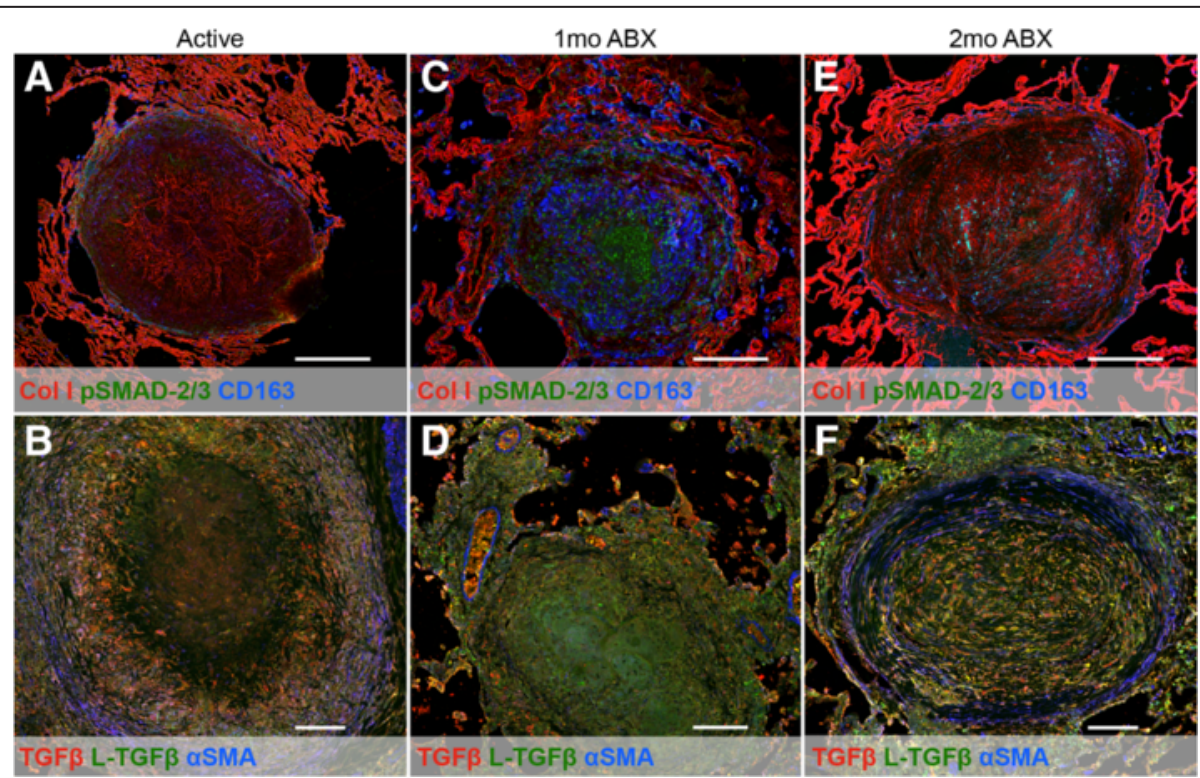

Fig. 3 Tuberculous granulomas bear signs of TGF $\beta$-driven fibrosis. Top panels feature granulomas stained for collagen I (red), phosphorylated SMAD-2/3 (green), and CD163 (blue). Bottom panels feature granulomas stained for TGF $\beta$ (red), L-TGF $\beta$ (green), and aSMA (blue). Magnification $\times 200$. Scale bar represents $500 \mu \mathrm{m}$. a, b Granulomas from animals with active disease. c, d Granulomas from animals after 1 month of TB chemotherapy. e, $\mathbf{f}$ Granulomas from animals after 2 months of TB chemotherapy 
portion of the lesion (Fig. 3b), an area populated by epithelioid macrophages $[5,43]$. Latent TGF $\beta$ was observed throughout this granuloma, and $\alpha \mathrm{SMA}$ was strongly present in a region of robust peripheral fibrosis. After 1 month of drug treatment, collagen I was detected around the edge of the structure, suggesting mild peripheral fibrotic development (Fig. 3c). Strong pSMAD-2/3 staining was interspersed among the CD163+ cells that were localized towards the center of the structure, a position in which they are not normally found during active disease. Another granuloma from an animal with 1 month of drug therapy exhibited very little $\alpha S M A$ or active TGF $\beta$, except minor staining in the exterior and in an adjacent blood vessel (Fig. 3d). Granulomas from animals 2 months post-chemotherapy displayed plentiful collagen I staining, with both peripheral fibrosis and central fibrotic organization (Fig. 3e). In this particular lesion, pSMAD-2/3 and CD163 appear to co-localize in the fibrosis. We found that $\alpha \mathrm{SMA}$ is present in a ring around the periphery of the structure (Fig. 3f). The active and latent forms of TGF $\beta$ appear to be intermixed within the lesion. These data overall indicate that active TGF $\beta$ is present in these granulomas and drives expression of TGF $\beta$-regulated products including $\alpha$ SMA.
Active Mtb infection and disease activates TGF $\beta$ and suppresses SMAD-2/3 signaling

We quantified median pixel intensity (MPI) from a collection of granulomas to elucidate how latent and active TGF $\beta$, $\alpha$ SMA, pSMAD2/3, collagen, and CD163 expression change over the three time points we are examining. We found an initial decrease in latent TGF $\beta$ levels at the initiation of drug treatment, but unchanged levels between 1 and 2 months of drug therapy (Fig. 4a). In contrast, active TGF $\beta$ appeared to decline in animals over the full 2-month course of drug therapy (Fig. 4b), while the trend in $\alpha$ SMA expression was consistent with trend observed for latent TGF $\beta$ (Fig. 4c). Interestingly, levels of phosphorylated SMAD-2/3 were higher after 1 month of chemotherapy but then decreased by 2 months of antibiotics (Fig. 4d). Relative amounts of collagen I and CD163 do not change between following antibiotic treatment (Fig. 4e-f). These data further demonstrate the role that active TGF $\beta$ is playing in drug-promoted tissue repair.

We used immunologic and biochemical assays to confirm our immunohistochemical analysis of TGF $\beta$ and collagen. Consistent with our immunohistochemical data, enzyme-linked immunosorbent assays (ELISAs) for

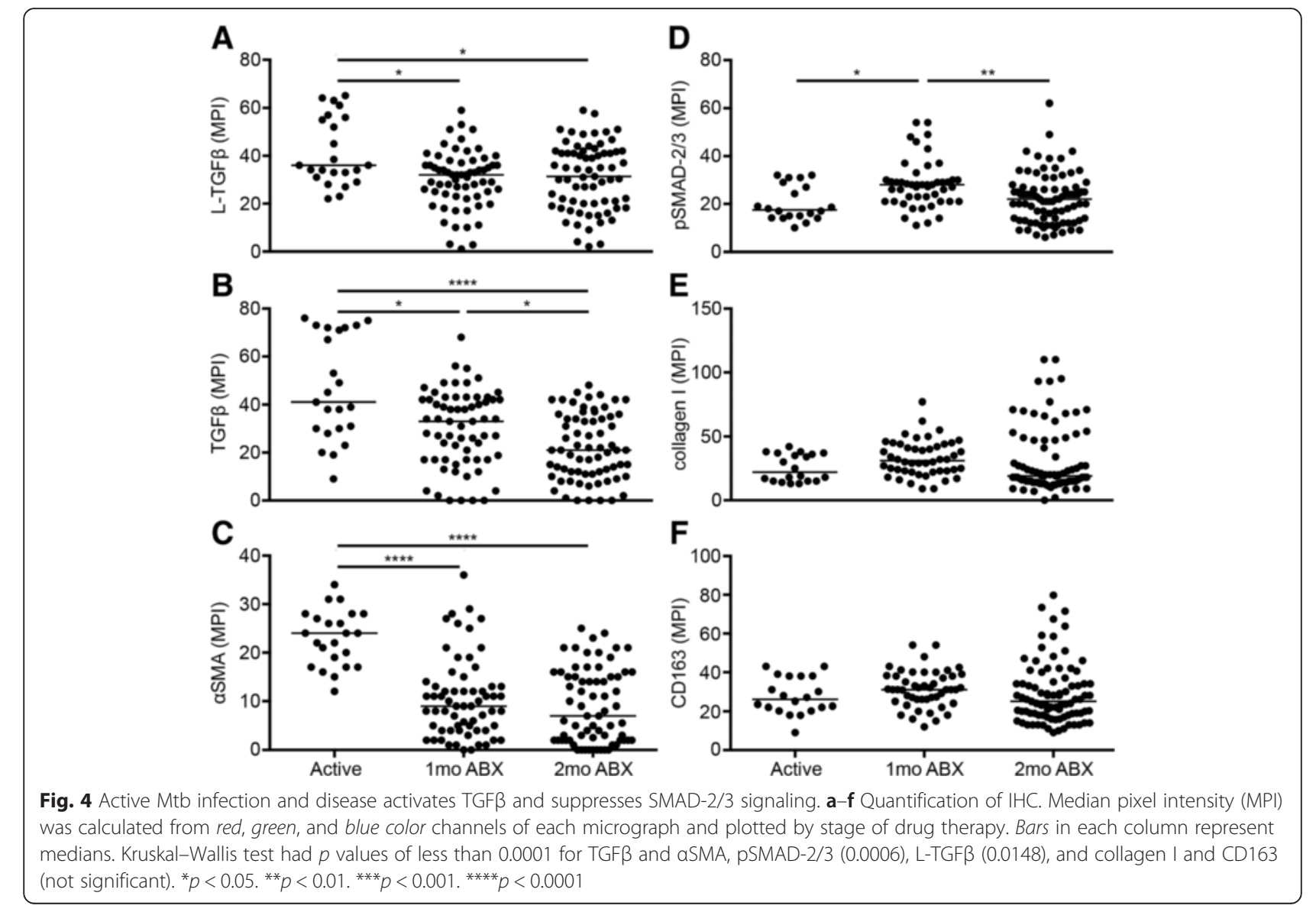


active TGF $\beta$ demonstrated greater quantities of TGF $\beta$ in granulomas from animals during active disease and lesser in animals after 2 months of drug treatment (Fig. 5a). Collagen levels were measured by hydroxyproline assay (Fig. 5b) and were unchanged between treated and untreated animals, coinciding with our microscopic data (Fig. 4f).

\section{TGF $\beta$ is strongly associated with collagen I expression}

We used our quantitative immunohistochemical data (Fig. 4) to perform pairwise correlations to identify the relationships between these factors. At all study time points, collagen I and pSMAD-2/3 exhibit a robust positive association (Fig. 6), especially during active disease and 1 month of drug therapy. In these time points, we found that over $50 \%$ of the variability in collagen I levels was explained by pSMAD-2/3 (Pearson's $r>0.7$ ). In granulomas from animals with active disease, or 2 months of drug treatment, there is a weak but significant association between pSMAD-2/3 and CD163 (Fig. 6). However, CD163 and collagen I are not statistically associated at any time point of the study (Fig. 6). This is in contrast to active TGF $\beta$, latent TGF $\beta$, and $\alpha \mathrm{SMA}$, which are all strongly and positively associated with one another at all time points (Additional file 3: Figure S3).

\section{Discussion}

The goal of this study was to identify molecular and cellular markers associated with fibrotic resolution in experimental tuberculosis infection. Tuberculosis produces a wide range of pulmonary pathologies through infection and disease, which typically result in chronic fibrocaseous disease [6, 7]. Drug treatment reduces bacterial burden and allows for tissue repair, although this does
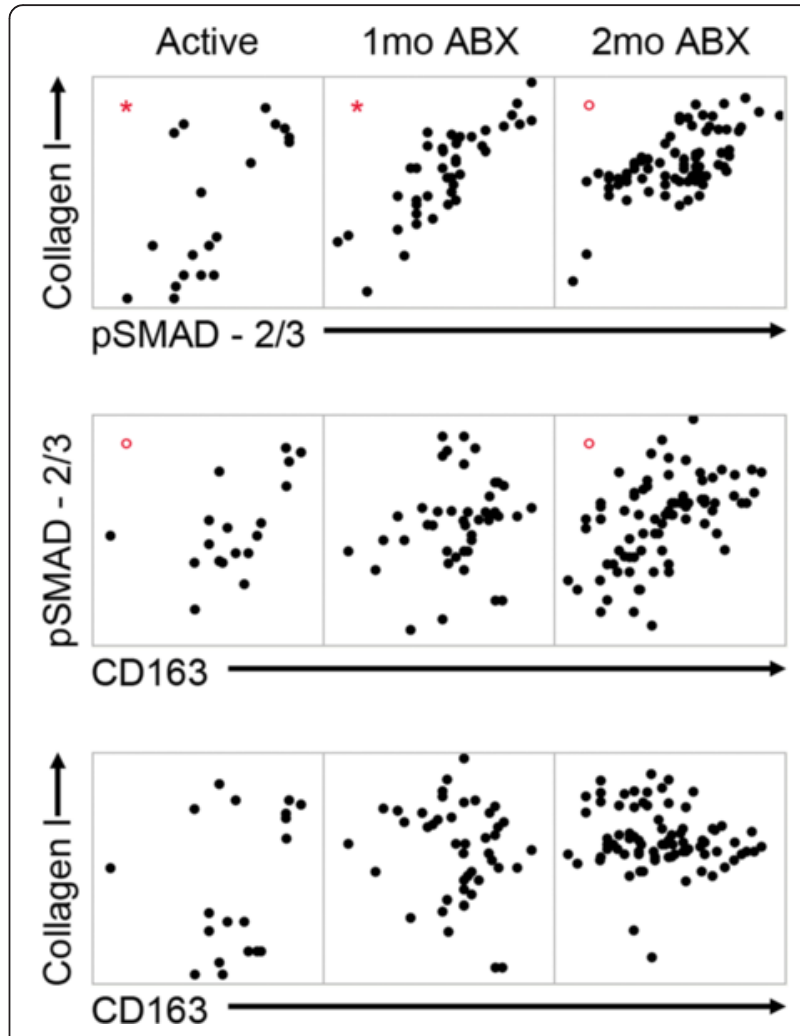

Fig. 6 TGF $\beta$ is strongly associated with the presence of collagen I. IHC values for each granuloma were plotted against one another to assess association between collagen I, TGF $\beta$ signaling, and M2 macrophages. Values were transformed, and Pearson's test for parametric correlation was used to determine significance. Significant correlations are denoted by red symbols. Open circles are significant but $r<0.7$, while asterisks indicate that $r>0.7$
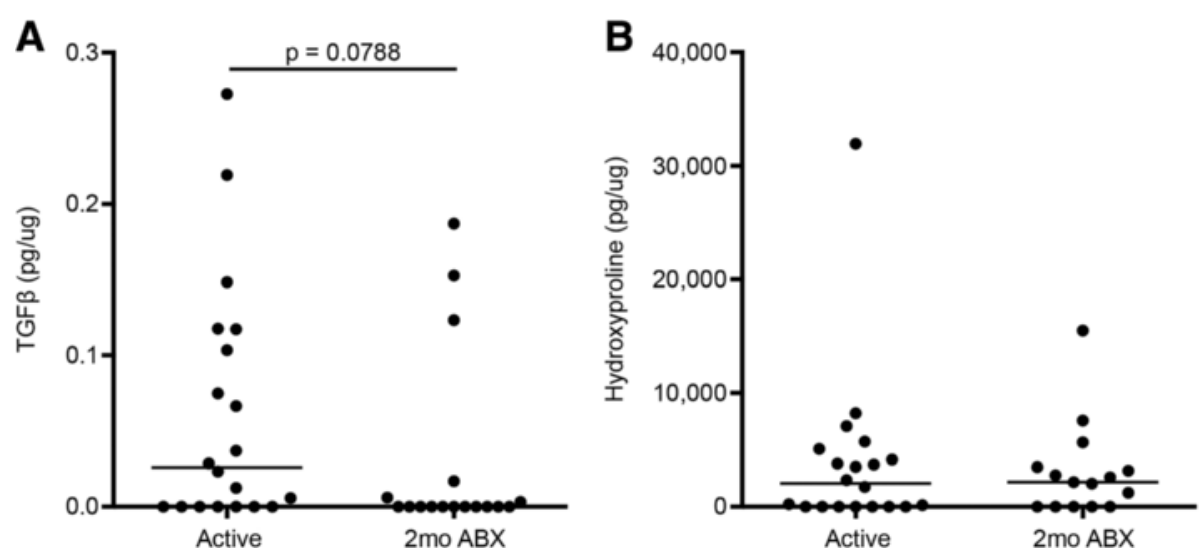

Fig. 5 Biochemical evidence of TGF $\beta$ and collagen. a ELISA for active TGF $\beta$. TGF $\beta$ in granulomas was converted into pg and then normalized by the total amount of protein in each granuloma as determined by BCA assay. Wilcoxon signed-rank test was used to compare granulomas from active disease to granulomas from 2 months of drug treatment. $\mathbf{b}$ Hydroxyproline assay. Collagen present in granulomas was determined using the hydroxyproline assay. Values were converted into pg and then normalized by the total amount of protein in each granuloma as determined by BCA assay 
not quickly alter the pathology remaining in the lung as demonstrated by computed tomography in both human and non-human primates [44]. Chemotherapy additionally leaves individuals vulnerable to relapse; more than half of smear positive TB cases have been previously treated for TB [45]. Relapse was originally thought to be cause by endogenous sources of Mtb remaining after drug treatment, but $75 \%$ of relapse cases in a high TB burden cohort were exogenously reinfected [46]. Why is this population prone to reinfection after successful chemotherapy? The risk of developing active TB is about 4.5 times higher in patients with chronic interstitial lung disease and idiopathic pulmonary fibrosis $[47,48]$. This could be due to the specific pathology of fibrotic lung tissue as it may promote colonization and establishment of infection [49]. The residual scar tissue left after drug therapy and loss of normal lung architecture could play roles in increasing the risk of relapse. Therefore, understanding the mechanism of fibrogenesis in $\mathrm{TB}$ and ameliorating tissue damage after drug treatment could reduce the risk of reinfection and help shrink the pool of infected individuals.

Several cell types are likely to play significant roles in driving fibrotic processes in granulomas, but it has been difficult to identify the specific cells that are the most important drivers of fibrosis. Histologic assessment can be used to evaluate the cellular responses to infection from a morphological perspective and can provide insights into lesion development and resolution. Identifying the cellular origin of some spindle-type cells in granulomas is not always possible, but histologic examination can provide important inferences as to whether these cells arose from epithelioid macrophages or collagen-producing fibroblasts. Based on our histologic observations and immunohistochemical analyses, we hypothesize that collagen-producing fibroblast-like cells may originate from several sources, and in some cases not from fibroblasts recruited from nearby tissues, but from non-fibroblast-like cells that have undergone a process similar to endothelial-mesenchymal progression. Additionally, circulating monocyte-derived cells (fibrocytes) can have phenotypes reminiscent of the phenotypes we have identified [50,51]. Given the ability of some circulating monocytes to differentiate into fibroblast-like cells instead of macrophages, the question of whether subsequent differentiation between macrophages and fibroblasts can occur should be considered. The wide range in collagen presence and the appearance of various collagenous phenotypes suggest that different mechanisms for fibrotic resolution occur in tuberculous granulomas. Some granulomas manifest both peripheral and central fibrous organization, while others demonstrate only central collagenization, or none at all. Future studies should seek to correlate these divergent repair mechanisms to lesion- specific Mtb burden to determine efficacy of clearance and elucidate the immunologic processes of each.

Our observations that latent TGF $\beta$ changes only slightly between granulomas from animals with active disease and drug-treated animals suggest that a pool of latent TGF $\beta$ remains in the lung even after Mtb is cleared. Latent TGF $\beta$ is maintained in the lung epithelium by latent TGF $\beta$-binding proteins where it can remain until activated [52]. Our data indicate that conditions within granulomas activate TGF $\beta$, an anti-inflammatory cytokine associated with fibrosis (Fig. 7). This molecule exerts biological action in the granuloma, as is supported by the presence of $\alpha$ SMA in direct proportion to TGF $\beta$. The lower levels of TGF $\beta$ and $\alpha$ SMA in granulomas of animals on drug therapy suggest that therapy itself does not activate more TGF $\beta$ and that the amount activated during active TB is utilized for tissue repair. Interestingly, phosphorylated SMAD-2/3 is lower during active disease, even though active TGF $\beta$ levels are high. Since SMAD-2/3 is phosphorylated when cells come into contact with active TGF $\beta$ [20], this suggests that live Mtb somehow blocks SMAD-2/3 activation even in the presence of active TGF $\beta$. The presence of $\alpha \mathrm{SMA}$, also an indicator of the cellular effects of TGF $\beta$, could be explained by the utilization of alternative TGF $\beta$-stimulated pathways $[53,54]$. The alternative TGF $\beta$ signaling pathways may be prominent in disease, and since the SMAD-2/3 pathway only becomes used in tissue repair once Mtb is cleared, the SMAD-2/3 pathway may be preferential for healing and clearance and the alternative pathways preferential for TB pathogenesis. Collagen I levels are similar between granulomas with active disease and drug treatment, suggesting that treatment only promotes and does not exacerbate fibrosis. Collagen I is strongly associated with active TGF $\beta$; over $50 \%$ of the variability in levels of collagen I is explained by TGF $\beta$ signaling through SMAD-2/3, indicating that active TGF $\beta$ is likely the main molecular driver of fibrosis and tissue repair in tuberculous lesions. CD163 and collagen I are not associated in this model, implying that the CD163+ macrophages are not major producers of collagen I. Macrophages in granulomas are not a uniform population of cells [5], and other CD163- macrophage subsets could be responsible for collagen production in the granuloma. Unfortunately, there are no pan-macrophage markers for primates, and our efforts to identify the specific population of cells responsible for collagen expression are ongoing [5]. Collagen appears to stream from the epithelioid macrophages into the caseous necrotic center. Interestingly, this population of cells is not comprised of the M2polarized CD163+ macrophages we expected would correlate with fibrosis, but instead appear to be M1-polarized cells [5], suggesting the capacity of these cells may be more diverse than previously known. Active TGF $\beta$ was mostly localized in the epithelioid macrophage region of 


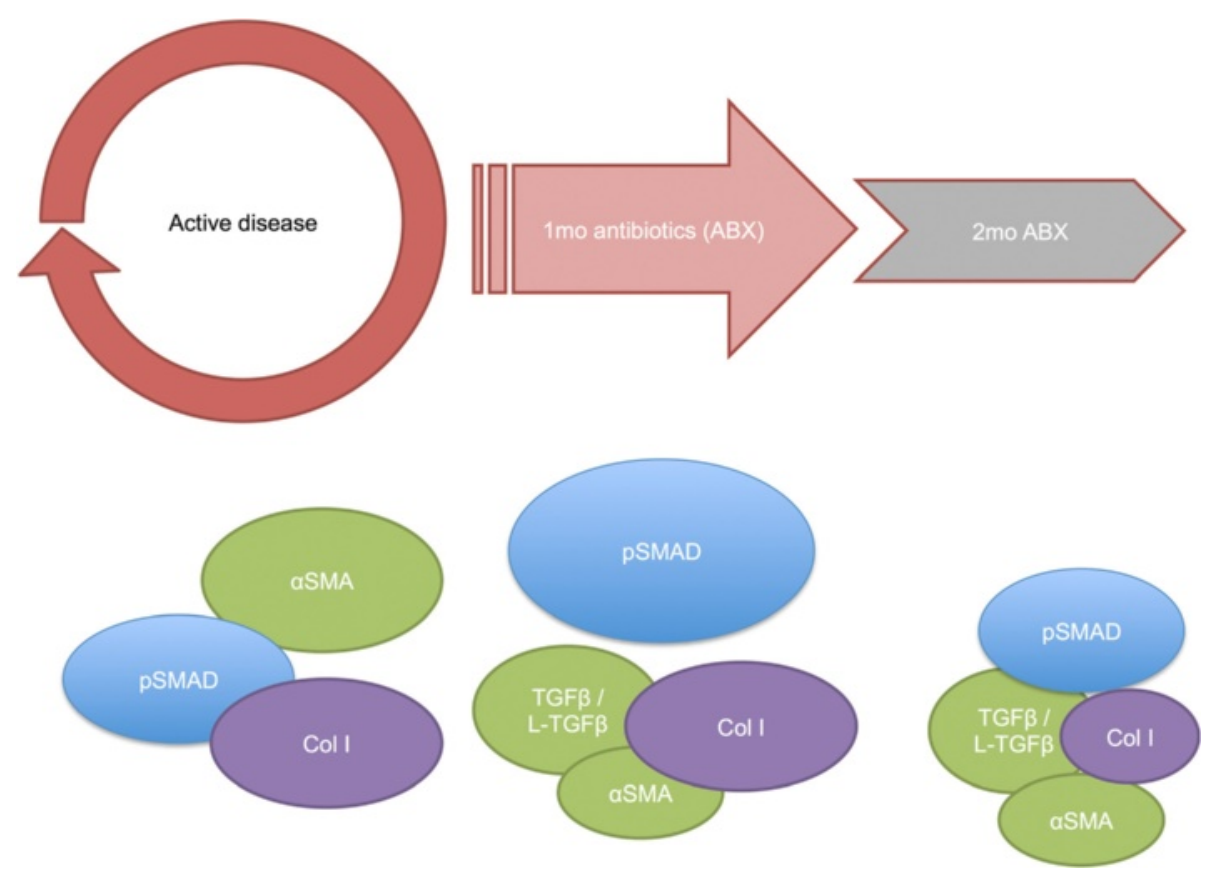

Fig. 7 Model that demonstrates the TGF $\beta$-associated processes explores in this study. The red arrow around active disease represents processes that activate fibrotic cytokines, which then diminish during the early course of drug therapy (smaller arrows that are less red). aSMA and TGF $\beta$ drop, represented in green, while pSMAD-2/3 appears to rise and fall, in blue. Levels of collagen I do not change, shown in purple

the granulomas as well. Alternatively, macrophageexpressed products could support collagen production by neighboring fibroblasts. These cells could potentially be contributors to collagenization, as well as surrounding lung epithelial cells transformed by active TGF $\beta$. Further work will include markers for these types of cells and determine their association with collagen production in the granuloma.

\section{Conclusions}

Fibrosis occurs in TB lesions during and after drug treatment. The goal of this project was to determine the cell types and molecules associated with fibrosis in nonhuman primates with TB. We provide additional evidence of activated TGF $\beta$ being present in lesions from $M$. tuberculosis-infected lung tissue. Collagen production in active disease and TB chemotherapy is strongly associated with TGF $\beta$, suggesting its role as the chief cytokine driving tissue repair. Future studies will seek to further explore these results mechanistically with hopes of developing adjunct treatment to minimize, or possibly reverse, scar formation after TB chemotherapy.

\section{Methods}

\section{Animals}

The Institutional Animal Care and Use Committee of University of Pittsburgh approved all experiments. The animals were housed and maintained in accordance with standards established in the Animal Welfare Act and the Guide for the Care and Use of Laboratory Animals.

\section{Infections}

Cynomolgus macaques (4-9 years of age) imported from China (Valley Biosystems, Sacramento, CA) were used for these studies $(n=21)$. Monkeys were infected via bronchoscope with $25 \mathrm{CFU}$ of $M$. tuberculosis Erdman strain. Using published criteria, monkeys were determined to have active or latent TB by $6-8$ months postinfection and were randomized to treatment or nontreatment groups; treatment was initiated when monkeys developed active TB, as determined by clinical and microbiologic signs $[43,55]$.

\section{Drug therapy}

The samples described in the current study were obtained from macaques in a previously published study from our laboratory [40,42]. Monkeys with active disease were as follows: untreated ( $n=9,110$ granulomas), treated with isoniazid (INH) and rifampin (RIF) for 1 month $(n=2,22$ granulomas) or 2 months $(n=5,91$ granulomas), or treated with INH, RIF, and metronidazole (MTZ) for 1 month ( $n=3,154$ granulomas) or 2 months $(n=5,93$ granulomas).

Necropsies were performed as previously described [35, 51]. Individual granuloma and lung samples were taken from each monkey from sites of infection and surrounding 
tissue. Portions of these samples were homogenized into single cell suspension before storing at $-80^{\circ} \mathrm{C}$ for ELISA and hydroxyproline assays, while another portion was formalin-fixed paraffin-embedded for histology.

\section{H\&E staining}

Formalin-fixed paraffin-embedded tissue sections were cut and stained with Harris hematoxylin modified (Sigma-Aldrich, St. Louis, MO) and eosin Y solution (Sigma-Aldrich, St. Louis, MO). Slides were deparaffinized in deionized water. Slides were then stained with hematoxylin for $3 \mathrm{~min}$. Slides were rinsed under running tap water, rinsed with $70 \%$ ethanol, and then stained with eosin for $3 \mathrm{~min}$. Slides were rinsed and dehydrated in ethanol, cleared in xylene, and then mounted. Criteria for characterizing granulomas were based on size and shape, type of granuloma, and cellular composition. A veterinary pathologist who is an expert in macaque tuberculosis (ECK) performed all histologic analyses.

\section{Masson's trichrome}

Formalin-fixed paraffin-embedded tissue sections were stained with Masson's trichrome to identify connective tissue, muscle, and collagen fibers (Sigma-Aldrich, St. Louis, MO). Slides were deparaffinized to deionized water. Slides were then immersed in Bouin's solution overnight at room temperature to intensify the subsequent staining. Slides were washed with tap water then stained with Harris hematoxylin solution (Sigma-Aldrich, St. Louis, MO) for $5 \mathrm{~min}$. Slides were washed again in running tap water for $5 \mathrm{~min}$, rinsed in deionized water, and stained in Biebrich scarlet-acid fuchsin for $5 \mathrm{~min}$. Slides were rinsed in deionized water and placed in phosphotungstic and phosphomolybdic acid solution for $5 \mathrm{~min}$. Slides were moved to Aniline Blue solution for $5 \mathrm{~min}$ and then placed in $1 \%$ acetic acid solution for $2 \mathrm{~min}$. Slides were rinsed in deionized water, dehydrated through alcohol, cleared in xylene, and then mounted.

\section{IHC}

Tissue sections were stained for collagen I (rabbit polyclonal, Abcam, Cambridge, MA, 1:50 dilution), pSMAD-2/3 (goat polyclonal, Santa Cruz Biotechnology, Dallas, TX, 1:10 dilution), CD163 (mouse clone 10D6, Neomarkers, Fremont, CA, 1:30 dilution), LTGF $\beta$ (goat polyclonal, R\&D Systems, Minneapolis, MN, 1:10 dilution), TGF $\beta$ (chicken polyclonal, R\&D Systems, Minneapolis, MN, 1:10 dilution), and $\alpha$ SMA (mouse clone 1A4, Thermo Fisher, Pittsburgh, PA, 1:100 dilution). Antigen retrieval and staining were done as previously described [5]. Briefly, formalin-fixed paraffin-embedded tissues samples were deparaffinized in xylene and rehydrated in ethanol. Samples were then placed into a pressure cooker with boiling antigen retrieval buffer (Tris-HCl, EDTA, Tween-20) for $7 \mathrm{~min}$. After allowing for the slides to cool, the sections were blocked with $2 \%$ fetal bovine serum in phosphatebuffered saline for $30 \mathrm{~min}$. Antibodies and fluorescent tags were incubated on each sample for $1 \mathrm{~h}$ with washes in between with phosphate-buffered saline with $0.2 \%$ Tween-20. Prolong Gold Mounting Medium with DAPI (Invitrogen) was then applied to the slides, which were then cured in the dark overnight before being imaged.

\section{Quantification of histology}

Trichrome- and H\&E-stained sections were imaged using Provis fluorescent microscope (Olympus America, Center Valley, PA) and fluorescently stained slides visualized with a FluoView 1000 confocal microscopes (Olympus). For images used for quantitative imaging, care was taken to keep the camera settings constant between granulomas and animals. These images were then saved as 24-bit TIFF files and read into the language $\mathrm{R}$ via the package "EBImage" from Bioconductor (http://www.bioconductor.org/packages/release/bioc/html/ EBImage.html). For the trichrome slides, the blue channel only was isolated. Red, green, and blue channels were pulled from the immunohistochemistry (IHC) slides. The median pixel intensity for each channel was then saved and exported to Microsoft Excel (Microsoft, Redmond, WA).

\section{ELISAs}

Active TGF $\beta$-1 in granuloma homogenates was measured by using a commercial ELISA (eBioscience, San Diego, CA) according to the manufacturer's instructions. Briefly, a high-affinity protein-binding plate was coated with a capture antibody overnight and blocked with assay diluent for an hour before adding standards and undiluted granuloma homogenates for overnight incubation. TGF $\beta$ was detected the next day using a biotinylated detection antibody and streptavidin-HRP, and the absorbance was immediately measured at $450 \mathrm{~nm}$. Total protein of the same samples was quantified by Pierce BCA Protein Assay (Thermo Scientific, Pittsburgh, PA), where granuloma homogenates were added to BCA Working Reagent, and the absorbance measured at $562 \mathrm{~nm}$ after $30 \mathrm{~min}$ at $37{ }^{\circ} \mathrm{C}$. Levels of TGF $\beta$ were normalized to total protein in granuloma homogenates.

\section{Hydroxyproline assay}

Collagen was detected in homogenized granulomas by using a commercial hydroxyproline kit (Sigma-Aldrich, St. Louis, MO). Briefly, samples were mixed with hydrochloric acid and hydrolyzed at $120{ }^{\circ} \mathrm{C}$ for $3 \mathrm{~h}$. These samples and standards were then transferred to a 96well plate and dried. Chloramine T/oxidation buffer was added to wells and incubated at room temperature for 
5 min. To this, diluted DMAB reagent was added and incubated at $60{ }^{\circ} \mathrm{C}$ for $90 \mathrm{~min}$. Absorbance at $560 \mathrm{~nm}$ was then measured. Levels of collagen were normalized to total protein in granuloma homogenates.

\section{Statistics}

Quantitative data from Masson's trichrome staining and immunohistochemical staining were visualized using Prism (Graphpad, La Jolla, CA). Analysis of these data was done using the tests indicated, typically a one-way ANOVA and a multiple comparison test for either parametric or non-parametric data. $p$ values were significant if less than 0.05. Further analysis of the immunohistochemical staining was performed with JMP (SAS, Cary, NC). Correlations between markers in histological stains were performed using the multivariate function, and scatter plots were generated. Significance was determined using pairwise correlations with the strength of the relationship given as Pearson's $r$.

\section{Additional files}

Additional file 1: Figure S1. Antibacterial chemotherapy promotes collagen-associated bacterial clearance. a The number of lung granulomas with viable bacterial growth were divided by the total number of lung granulomas and plotted for each animal in the three groups. Each dot represents one animal. Bars represent medians. $\mathbf{b}$ The median of the median pixel intensity of the aniline blue staining was determined. This was then plotted against the percent of lung granulomas with viable bacteria. Significance of the interaction between percent of Mtb + lung granulomas and collagen staining $(p)$ and strength of interaction $\left(R^{2}\right)$ are shown. Each dot represents one animal, and colors match the colors of the treatment groups in part A of this figure. Dotted lines represent $95 \%$ confidence intervals. (TIF $271 \mathrm{~kb}$ )

Additional file 2: Figure S2. Negative and positive controls for immunohistochemistry. Left panels feature negative controls for $\mathrm{HC}$ and right panels feature blood vessels, which should serve as bright signals for most of the markers. Negative controls did not receive primary antibodies, but received secondary antibodies and were imaged using the same settings as the slides for the study. $\mathbf{a}-\mathbf{b}$ Negative control and blood vessel for the first panel. c-d Negative control and blood vessel using the second panel. (TIF $7053 \mathrm{~kb}$ )

Additional file 3: Figure S3. Active TGF $\beta$ is highly associated with its latent form and ASMA. IHC values for each granuloma were plotted against one another to assess association between TGF $\beta$, L-TGF $\beta$, and aSMA — which is produced by cells activated by TGF $\beta$. Values were transformed, and Pearson's test for parametric correlation was used to determine significance. Significant correlations are denoted by red symbols. Open circles are significant but $r<0.7$, while asterisks indicate that $r>0.7$. (TIF $843 \mathrm{~kb}$ )

\section{Abbreviations}

ABX: antibiotics; H\&E: hematoxylin and eosin; IHC: immunohistochemistry; INH: isoniazid; Mtb: Mycobacterium tuberculosis; MTZ: metronidazole; RIF: rifampicin; TB: tuberculosis; TGF $\beta$ : transforming growth factor- $\beta$; aSMA: a-smooth muscle actin.

\section{Competing interests}

The authors declare that they have no competing interests.

\section{Authors' contributions}

RMD completed the histological staining and related microscopy (except for Fig. 1 which was completed by ECK), quantified the histological staining and immunohistochemical staining, and performed all statistics. RMD, JTM, and $\mathrm{MH}$ carried out the immunohistochemical staining and related microscopy. JTM, LRC, and EAW performed the ELISAs. JTM and LRC carried out the hydroxyproline assays. RMD, JTM, and JLF conceived the study, participated in its design and coordination, and these authors in addition to ECK helped to draft the manuscript. All authors read and approved the final manuscript.

\section{Acknowledgements}

We would like to thank the members of the Flynn lab for their support and constructive criticisms, especially Pauline Maiello for the statistical queries and Chelsea Chedrick for the figure preparation. We would also like to thank the Center for Biologic Imaging and the Department of Microbiology and Molecular Genetics at the University of Pittsburgh School of Medicine for the use of their Provis light microscope and Fluoview 1000 confocal microscope, respectively. This study was funded by NIH HL110811.

\section{Author details}

${ }^{1}$ Department of Microbiology and Molecular Genetics, University of Pittsburgh School of Medicine, Pittsburgh, PA 15261, USA. ²Department of Infectious Diseases and Microbiology, Pitt Public Health, University of Pittsburgh, Pittsburgh, PA 15261, USA. ${ }^{3}$ Division of Laboratory Animal Resources, University of Pittsburgh, Pittsburgh, PA 15261, USA. ${ }^{4}$ University of Pittsburgh School of Pharmacy, Pittsburgh, PA 15261, USA.

Received: 13 January 2016 Accepted: 25 April 2016

Published online: 27 April 2016

\section{References}

1. WHO. Global tuberculosis report. 2014

2. Ramakrishnan L. Revisiting the role of the granuloma in tuberculosis. Nat Rev Immunol. 2012;12(5):352-66.

3. Russell DG, Barry 3rd CE, Flynn JL. Tuberculosis: what we don't know can, and does, hurt us. Science. 2010;328(5980):852-6.

4. Russell DG. Who puts the tubercle in tuberculosis? Nat Rev Microbiol. 2007; 5(1):39-47.

5. Mattila JT, Ojo OO, Kepka-Lenhart D, Marino S, Kim JH, Eum SY, et al. Microenvironments in tuberculous granulomas are delineated by distinct populations of macrophage subsets and expression of nitric oxide synthase and arginase isoforms. J Immunol. 2013;191(2):773-84.

6. Hunter RL. Pathology of post primary tuberculosis of the lung: an illustrated critical review. Tuberculosis (Edinb). 2011;91(6):497-509.

7. Canetti G. The tubercle bacillus in the pulmonary lesion of man. New York: Springer; 1955. p. 230

8. Lin PL, Ford CB, Coleman MT, Myers AJ, Gawande R, loerger T, et al. Sterilization of granulomas is common in active and latent tuberculosis despite within-host variability in bacterial killing. Nat Med. 2014;20(1):75-9.

9. Cutroneo KR. How is type I procollagen synthesis regulated at the gene level during tissue fibrosis. J Cell Biochem. 2003;90(1):1-5.

10. Sime PJ, O'Reilly KM. Fibrosis of the lung and other tissues: new concepts in pathogenesis and treatment. Clin Immunol. 2001;99(3):308-19.

11. Wynn TA. Integrating mechanisms of pulmonary fibrosis. J Exp Med. 2011; 208(7):1339-50.

12. Wynn TA, Ramalingam TR. Mechanisms of fibrosis: therapeutic translation for fibrotic disease. Nat Med. 2012;18(7):1028-40.

13. Mi S, Li Z, Yang HZ, Liu H, Wang JP, Ma YG, et al. Blocking IL-17A promotes the resolution of pulmonary inflammation and fibrosis via TGF-beta1dependent and -independent mechanisms. J Immunol. 2011;187(6):3003-14.

14. Sun L, Louie MC, Vannella KM, Wilke CA, LeVine AM, Moore BB, et al. New concepts of IL-10-induced lung fibrosis: fibrocyte recruitment and M2 activation in a CCL2/CCR2 axis. Am J Physiol Lung Cell Mol Physiol. 2011; 300(3):L341-53.

15. Redente EF, Keith RC, Janssen W, Henson PM, Ortiz LA, Downey GP, et al. Tumor necrosis factor-alpha accelerates the resolution of established pulmonary fibrosis in mice by targeting profibrotic lung macrophages. Am J Respir Cell Mol Biol. 2014;50(4):825-37.

16. Fielding CA, Jones GW, McLoughlin RM, McLeod L, Hammond VJ, Uceda J, et al. Interleukin-6 signaling drives fibrosis in unresolved inflammation. Immunity. 2014;40(1):40-50.

17. Gistera A, Robertson AK, Andersson J, Ketelhuth DF, Ovchinnikova O, Nilsson SK, et al. Transforming growth factor-beta signaling in T cells promotes 
stabilization of atherosclerotic plaques through an interleukin-17-dependent pathway. Sci Transl Med. 2013;5(196):196ra00.

18. Lee CG, Homer RJ, Zhu Z, Lanone S, Wang X, Koteliansky V, et al. Interleukin-13 induces tissue fibrosis by selectively stimulating and activating transforming growth factor beta(1). J Exp Med. 2001;194(6):809-21.

19. Khalil N. TGF-beta: from latent to active. Microbes Infect. 1999;1(15):1255-63.

20. Massague J. TGF-beta signal transduction. Annu Rev Biochem. 1998;67:753-91.

21. Broekelmann TJ, Limper AH, Colby TV, McDonald JA. Transforming growth factor $B 1$ is present at sites of extracellular matrix gene expression in human pulmonary fibrosis. Proc Natl Acad Sci U S A. 1991;88(15):6642-6.

22. Khalil N, Bereznay O, Sporn M, Greenberg AH. Macrophage production of transforming growth factor beta and fibroblast collagen synthesis in chronic pulmonary inflammation. J Exp Med. 1989;170(3):727-37.

23. Limper AH, Colby TV, Sanders MS, Asakura S, Roche PC, DeRemee RA. Immunohistochemical localization of transforming growth factor-beta 1 in the nonnecrotizing granulomas of pulmonary sarcoidosis. Am J Respir Crit Care Med. 1994;149(1):197-204

24. Khalil N, Parekh TV, O'Connor R, Antman N, Kepron W, Yehualaeshet T, et al. Regulation of the effects of TGF-beta1 by activation of latent TGF-beta1 and differential expression of TGF-beta receptors (TbetaR-I and TbetaR-II) in idiopathic pulmonary fibrosis. Thorax. 2001;56:907-15.

25. Lekkerkerker AN, Aarbiou J, van Es T, Janssen RAJ. Cellular players in lung fibrosis. Curr Pharm Des. 2012;18:4093-102.

26. Pechkovsky DV, Prasse A, Kollert F, Engel KM, Dentler J, Luttmann W, et al. Alternatively activated alveolar macrophages in pulmonary fibrosismediator production and intracellular signal transduction. Clin Immunol. 2010;137(1):89-101.

27. Pasipanodya JG, Miller TL, Vecino M, Munguia G, Garmon R, Bae S, et al. Pulmonary impairment after tuberculosis. Chest. 2007;131(6):1817-24.

28. Pasipanodya J, McNabb SJN, Hilsenrath P, Bae S, Lykens K, Vecino E, et al. Pulmonary impairment after tuberculosis and its contribution to TB burden. BMC Public Health. 2010;10:259.

29. Hnizdo E, Singh T, Churchyard G. Chronic pulmonary function impairment caused by initial and recurrent pulomary tuberculosis following treatment. Thorax. 2000;55:32-8.

30. Bansal V, Prasad R. Pulmonary rehabilitation in chronic respiratory diseases. Indian J Chest Dis Allied Sci. 2014;56:147-8.

31. Dheda K, Booth H, Huggett JF, Johnson MA, Zumla A, Rook GA. Lung remodeling in pulmonary tuberculosis. J Infect Dis. 2005;192(7):1201-9.

32. Via LE, Lin PL, Ray SM, Carrillo J, Allen SS, Eum SY, et al. Tuberculous granulomas are hypoxic in guinea pigs, rabbits, and nonhuman primates. Infect Immun. 2008;76(6):2333-40.

33. Ong CW, Elkington PT, Friedland JS. Tuberculosis, pulmonary cavitation, and matrix metalloproteinases. Am J Respir Crit Care Med. 2014;190(1):9-18.

34. Marshall BG, Wangoo A, Cook HT, Shaw RJ. Increased inflammatory cytokines and new collagen formation in cutaneous tuberculosis and sarcoidosis. Thorax. 1996;51:1253-61.

35. Toossi Z, Young TG, Averill LE, Hamilton BD, Shiratsuchi H, Ellner JJ. Induction of transforming growth factor beta 1 by purified protein derivative of Mycobacterium tuberculosis. Infect Immun. 1995;63(1):224-8.

36. Aung $\mathrm{H}, \mathrm{Wu} \mathrm{M}$, Johnson $\mathrm{L}$, Hirsch CS, Toossi Z. Bioactivation of latent transforming growth factor beta1 by Mycobacterium tuberculosis in human mononuclear phagocytes. Scand J Immunol. 2005;61(6):558-65.

37. Aung $H$, Toossi Z, McKenna SM, Gogate P, Sierra J, Sada E, et al. Expression of transforming growth factor- $\beta$ but not tumor necrosis factor- $a$, interferon$\gamma$, and interleukin-4 in granulomatous lung lesions in tuberculosis. Tuber Lung Dis. 2000;80(2):61-7.

38. Shin HJ, Kwon YS. Treatment of drug susceptible pulmonary tuberculosis. Tuberc Respir Dis (Seoul). 2015;78(3):161-7.

39. Dheda K, Barry CE, Maartens G. Tuberculosis. The Lancet. 2016;387(10024): 1211-26. Pubmed ID: 26377143.

40. Lin PL, Dartois V, Johnston PJ, Janssen C, Via L, Goodwin MB, et al. Metronidazole prevents reactivation of latent Mycobacterium tuberculosis infection in macaques. Proc Natl Acad Sci U S A. 2012;109(35):14188-93.

41. Van Linthout S, Miteva K, Tschope C. Crosstalk between fibroblasts and inflammatory cells. Cardiovasc Res. 2014;102(2):258-69.

42. Lin PL, Coleman MT, Carney JP, Lopresti BJ, Tomko J, Fillmore D, et al. Radiologic responses in cynomolgus macaques for assessing tuberculosis chemotherapy regimens. Antimicrob Agents Chemother. 2013;57(9):4237.

43. Capuano 3rd SV, Croix DA, Pawar S, Zinovik A, Myers A, Lin PL, et al. Experimental Mycobacterium tuberculosis infection of cynomolgus macaques closely resembles the various manifestations of human $\mathrm{M}$. tuberculosis infection. Infect Immun. 2003;71(10):5831-44.

44. Coleman MT, Chen RY, Lee M, Lin PL, Dodd LE, Maiello P, et al. PET/CT imaging reveals a therapeutic response to oxazolidinones in macaques and humans with tuberculosis. Sci Transl Med. 2014;6(265):265ra167.

45. den Boon S, van Lill S, Borgdorff MW, Enarson DA, Verver S, Bateman ED, et al. High prevalence of tuberculosis in previously treated patients, Cape Town, South Africa. Emerg Infect Dis. 2007;13(8):1189-94.

46. van Rie A, Warren R, Richardson M, Victor TC, Gie RP, Enarson DA, et al. Exogenous reinfection as a cause of recurrent tuberculosis after curative treatment. N Engl J Med. 1999;341:1174-9.

47. Shachor Y, Schindler D, Siegal A, Lieberman D, Mikulski Y, Bruderman I. Increased incidence of pulmonary tuberculosis in chronic interstitial lung disease. Thorax. 1989;44(2):151-3.

48. Chung MJ, Goo JM, Im JG. Pulmonary tuberculosis in patients with idiopathic pulmonary fibrosis. Eur J Radiol. 2004;52(2):175-9.

49. Ahn $\mathrm{CH}$, Nash DR, Hurst GA. Ventilatory defects in atypical mycobacteriosis. Am Rev Respir Dis. 1976;113(3):273-9.

50. Bellini A, Mattoli S. The role of the fibrocyte, a bone marrow-derived mesenchymal progenitor, in reactive and reparative fibroses. Lab Invest. 2007;87(9):858-70.

51. Reilkoff RA, Bucala R, Herzog EL. Fibrocytes: emerging effector cells in chronic inflammation. Nat Rev Immunol. 2011;11(6):427-35.

52. Camelo A, Dunmore R, Sleeman MA, Clarke DL. The epithelium in idiopathic pulmonary fibrosis: breaking the barrier. Front Pharmacol. 2014;4:173.

53. Daniels CE, Wilkes MC, Edens M, Kottom TJ, Murphy SJ, Limper AH, et al. Imatinib mesylate inhibits the profibrogenic activity of TGF-beta and prevents bleomycin-mediated lung fibrosis. J Clin Invest. 2004;114(9):1308-16.

54. Hu Y, Peng J, Feng D, Chu L, Li X, Jin Z, et al. Role of extracellular signalregulated kinase, p38 kinase, and activator protein-1 in transforming growth factor-beta1-induced alpha smooth muscle actin expression in human fetal lung fibroblasts in vitro. Lung. 2006;184(1):33-42.

55. Lin PL, Rodgers M, Smith L, Bigbee M, Myers A, Bigbee C, et al. Quantitative comparison of active and latent tuberculosis in the cynomolgus macaque model. Infect Immun. 2009;77(10):4631-42.

\section{Submit your next manuscript to BioMed Central and we will help you at every step:}

- We accept pre-submission inquiries

- Our selector tool helps you to find the most relevant journal

- We provide round the clock customer support

- Convenient online submission

- Thorough peer review

- Inclusion in PubMed and all major indexing services

- Maximum visibility for your research

Submit your manuscript at www.biomedcentral.com/submit
C Biomed Central 